\title{
A computer program for Spearman-Kärber and probit analysis of psychometric function data
}

\author{
JEFF MILLER \\ University of Otago, Dunedin, New Zealand \\ and \\ ROLF ULRICH \\ University of Tübingen, Tübingen, Germany
}

\begin{abstract}
PMETRIC is a computer program for the analysis of observed psychometric functions. It can estimate the parameters of these functions, using either probit analysis (a parametric technique) or the Spearman-Kärber method (a nonparametric one). For probit analysis, either a maximum likelihood or a minimum $\chi^{2}$ criterion may be used for parameter estimation. In addition, standard errors of parameter estimates can be estimated via bootstrapping. The program can be used to analyze data obtained from either yes-no or $m$-alternative forced-choice tasks. To facilitate the use of PMETRIC in simulation work, an associated program, PMETGEN, is provided for the generation of simulated psychometric function data. Use of PMETRIC is illustrated with data from a duration discrimination task.
\end{abstract}

The psychometric function is an important tool in psychophysics. It can be used to measure absolute thresholds, points of subjective equality, and difference limens (DLs; see, e.g., Gescheider, 1997; Woodworth \& Schlosberg, 1954). Although signal detection theory also provides powerful tools for psychophysical measurement (see, e.g., Macmillan \& Creelman, 1991; Wickens, 2001), these have not replaced the more classical psychophysical approaches based on the analysis of psychometric functions (cf. Ernst \& Banks, 2002; Grondin, 2001b; Rammsayer \& Ulrich, 2001; Spence, Shore, \& Klein, 2001; for a review, see Gescheider, 1997). Indeed, a recent special issue of Perception \& Psychophysics, edited by Klein and Macmillan (2001), covered new developments in the estimation and analysis of psychometric functions.

There are several reasons why this classical approach remains worthwhile. (1) Psychometric functions are sometimes used to test specific psychophysical models that are more difficult to test with the tools provided by signal detection theory (see, e.g., Ernst \& Banks, 2002; Falmagne, 1985; Rammsayer \& Ulrich, 2001; Sternberg \& Knoll, 1973). (2) Adaptive procedures enjoy widespread use in psychophysicsbecause they increase the efficiency of estimating certain parameters of psychometric functions (cf. Kaernbach, 1991; Leek, 2001). To our knowledge, comparable adaptive procedures do not exist for measures based on signal detection theory. (3) Measure-

We acknowledge the helpful comments of two anonymous reviewers. Correspondence concerning this article should be addressed to J. Miller, Department of Psychology, University of Otago, Dunedin, New Zealand (e-mail: miller@psy.otago.ac.nz). ments of the DLs-unlike those of $d^{\prime}$-allow construction of sensation magnitude scales, such as the dol scale for the perception of pain (e.g., Marks \& Gescheider, 2002). (4) Finally, the median (i.e., point of subjective equality) of the psychometric function is often employed to assess the existence of a perceptual illusion (e.g., Flanagan \& Bandomir, 2000; Rose \& Summers, 1995).

An observed psychometric function plots the proportion of times a certain response is given as a function of some property of a physical stimulus. For example, Table 1 provides data obtained in a duration discrimination task by Grondin (2001a). In this task, an observer (Participant 2) was presented sequentially with a standard tone of duration $d_{\mathrm{s}}$ and a comparison tone of duration $d_{\mathrm{c}}$. The standard duration was always $900 \mathrm{msec}$, and the $10 \mathrm{com}-$ parison durations ranged from 630 to $1,170 \mathrm{msec}$. The duration of the comparison tone varied randomly from trial to trial, and each duration was presented a total of 120 times. The observer was asked to report whether each comparison tone was longer than the standard. Figure 1 depicts the psychometric function obtained for this observer, showing the observed probability of the comparison longer response as a function of the duration of the comparison tone. These data display the pattern typical of psychometric functions, where the probability of the measured response, plotted on the ordinate, increases with the stimulus value, plotted on the abscissa. This increase is usually monotonic when the number of observations per stimulus value is large. When this number is relatively small, however, observed psychometric functions are often nonmonotonic, due to binomial variability in the observed response probabilities.

The classical (e.g., Fechner, 1860) and, presumably, most common procedure for summarizing the data from 
Table 1

Observed $\boldsymbol{r}$ Responses as a Function of Comparison Duration

\begin{tabular}{|c|c|c|c|c|c|c|c|c|c|c|}
\hline \multirow[b]{2}{*}{ Summary Measure } & \multicolumn{10}{|c|}{ Duration of Comparison Stimulus (msec) } \\
\hline & 630 & 690 & 750 & 810 & 870 & 930 & 990 & 1,050 & 1,110 & 1,170 \\
\hline$N$ of $r$ responses, $X_{i}$ & 1 & 0 & 0 & 11 & 60 & 87 & 114 & 119 & 120 & 120 \\
\hline \multirow{2}{*}{$\begin{array}{l}N \text { of } \bar{r} \text { responses, } n_{i}-X_{i} \\
\hat{p}\end{array}$} & 119 & 120 & 120 & 109 & 60 & 23 & 6 & 1 & 0 & 0 \\
\hline & .01 & .00 & .00 & .09 & .50 & .79 & .95 & .99 & 1.0 & 1.0 \\
\hline \multicolumn{11}{|c|}{$\begin{array}{l}\text { Note-Results of an experiment in which an observer was presented with a standard tone with a duration of } \\
900 \mathrm{msec} \text {, followed by a comparison tone of } 1 \text { of the } 10 \text { durations indicated. Each comparison duration was } \\
\text { tested in } n_{i}=120 \text { trials. For each comparison duration, the observed numbers of comparison longer }(r) \text { and } \\
\text { comparison not longer }(\bar{r}) \text { responses, } X_{i} \text { and } n_{i}-X_{i} \text {, are tabulated, and the estimated probability of the } r \text { re- } \\
\text { sponse is } \hat{p} \text {. (We thank Simon Grondin for providing us with these data.) }\end{array}$} \\
\hline
\end{tabular}

an observed psychometric function is probit analysis (e.g., Finney, 1952, 1978). Probit analysis was originally developed for the analysis of data from toxicological assays (Bliss, 1935), and it is generally useful in examining the dependence of any binomial outcome variable (e.g., lives or dies) on any physical quantity (e.g., amount of poison). In the probit analysis of psychophysical data, the psychometric function is generally assumed to have the shape of a cumulative normal distribution, often referred to as a normal ogive. Given this assumption, the data are used to estimate the parameters of the normal distribution (i.e., $\mu$ and $\sigma$ ) via one of several alternative techniques (see, e.g., Finney, 1978; Foster \& Bischof, 1987).

In bioassay, for example, a researcher might want to determine the relationship between the dosage of a certain poison and the probability that a certain pest animal consuming that dose will die. In a typical study, each of $k$ different stimulus dosages, $s_{1}<s_{2} \ldots<s_{k}$, is given to

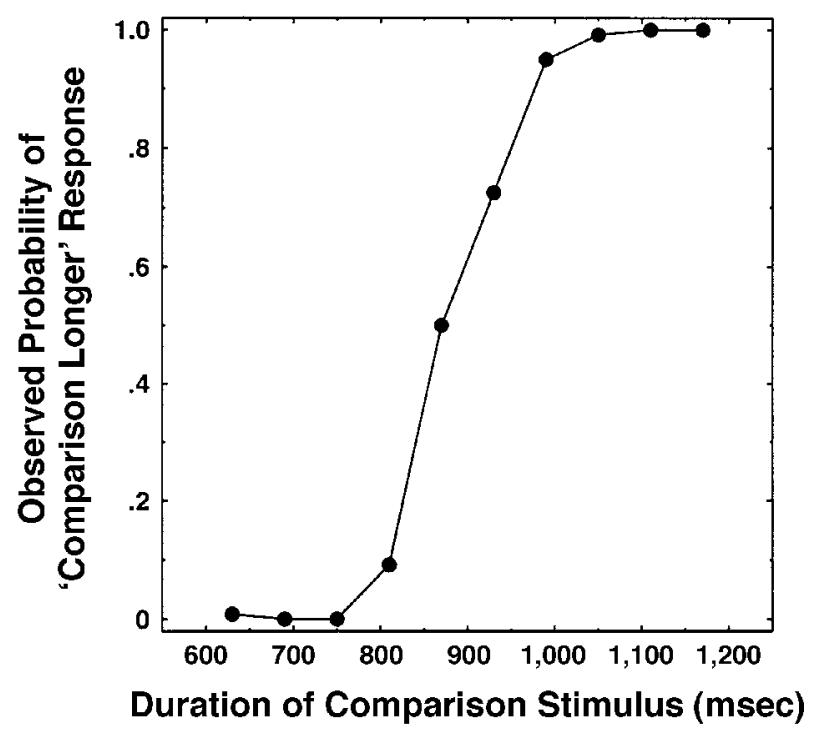

Figure 1. Psychometric function plotted from the duration discrimination data of Grondin (2001a) shown in Table 1 . The function shows the observed proportion of trials in which a comparison duration was judged to be longer than a standard duration as a function of the comparison duration. $n_{i}$ different animals $(i=1 \ldots k)$, and the number of deaths at each dosage $i$ is counted, $X_{i}$. Such data are typically analyzed with a statistical model in which it is assumed that each animal has a minimum lethal dosage and that the animal dies if and only if it is given a dosage greater than or equal to its minimum lethal dose. Thus, an observed $\hat{p}_{i}=X_{i} / n_{i}$ value is an estimate of the population proportion of animals for whom the lethal dose is less than or equal to $s_{i}$.

Although probit analysis provides a classical tool for summarizing an observed psychometric function, there are now several alternative approaches. For example, some authors prefer to fit a cumulative logistic or Weibull distribution (e.g., Strasburger, 2001a), because these distributions often provide superior fits to observed data (e.g., Strasburger, 2001b) and are sometimes of particular theoretical interest (e.g., Quick, 1974). Thus, the choice for such alternative shapes of the psychometric function may be guided by both theoretical and practical considerations.

In addition, in some cases, observed psychometric functions may be inconsistent with the assumption of any specific distribution considered by the researcher. For example, a $\chi^{2}$ test (Guilford, 1936) may indicate that a set of data is not in agreement with the assumption of the normal, logistic, or Weibull distribution (e.g., G. A. Miller \& Garner, 1944; Stevens, Morgan, \& Volkmann, 1941). In these situations, the so-called Spearman-Kärber method provides a distribution-free method for the estimation of psychometric functions (e.g., Epstein \& Churchman, 1944; Kärber, 1931; J. Miller \& Ulrich, 2001; Spearman, 1908; Sternberg, Knoll, \& Zukofsky, 1982).

In brief, like probit analysis, the Spearman-Kärber method treats the psychometric function observed in a yes-no task as a cumulative probability density function $(\mathrm{CDF})^{1}$ from which any desired percentiles or moments can be estimated. More specifically, the Spearman-Kärber method assumes that this CDF emerges from grouped data-an assumption that allows the reconstruction of the corresponding histogram. Suppose an experimenter employs $k$ stimulus values, $s_{1}<s_{2} \ldots<s_{k}$, to estimate the observed response probabilities, $\hat{p}_{i},(i=1 \ldots k)$, associated with each stimulus value. The estimated probability related to the stimulus interval (or bin) from $s_{i-1}$ to $s_{i}$ would then be equal to $\hat{p}_{i}-\hat{p}_{i-1}$. Assuming a uniform 
distribution within this bin, the probability density within the bin is then estimated by $\left(\hat{p}_{i}-\hat{p}_{i-1}\right) /\left(s_{i}-s_{i-1}\right)$. Thus, this reconstructed histogram approximates the probability density function associated with the CDF of the true psychometric function. It can be shown (Church \& Cobb, 1973; Sternberg et al., 1982, pp. 234-236) that the $r$ th raw moment of this histogram (and thus of the observed psychometric function) is given by

$$
\hat{\mu}_{r}=\frac{1}{r+1} \sum_{i=1}^{k+1} \frac{\left(\hat{p}_{i}-\hat{p}_{i-1}\right)\left(s_{i}^{r+1}-s_{i-1}^{r+1}\right)}{s_{i}-s_{i-1}} .
$$

(The values $s_{0}$ and $s_{k+1}$ are chosen such that one can assume $p_{0}=0$ and $p_{k+1}=1$.) Higher central momentsincluding the variance, skewness, and kurtosis of the psychometric function-can be directly computed from these raw moments. Simulations comparing the nonparametric Spearman-Kärber method with parametric approaches involving the normal, Weibull, and several other psychometric functions suggest that the SpearmanKärber method may be superior even when the underlying distributional assumption is satisfied (J. Miller \& Ulrich, 2001). In addition, Ulrich and Miller (in press) have shown how this nonparametric method can also be used to analyze data from $m$-alternative forced-choice ( $m$-AFC) tasks.

\section{The PMETRIC Program}

PMETRIC is a computer program for the analysis of observed psychometric functions. It is written in Pascal and should run on all IBM PC-compatible microcomputers under the Windows operating system. An OS/2 version is also available from the first author.

PMETRIC has two main features not present in previous programs for the analysis of psychometric functions (e.g., Lieberman, 1983). First, it provides an analysis of psychometric functions using the Spearman-Kärber method, in addition to any specified distributional analysis. We are not aware of any other statistical software (e.g., SPSS, SAS, or JMP) that includes this nonparametric option for analyzing psychometric functions. Second, PMETRIC allows the user to fit data with a greater range of alternative distributions (e.g., logistic, Weibull, rectangular, etc.) than does any other software of which we are aware.

PMETRIC reads data from an ASCII file containing the information shown in Table 1 . The first line of the file contains the stimulus values tested in the experiment (in this case, the 10 stimulus durations) plus assumed lower and upper bounds for the psychometric function (e.g., durations of 600 and 1,200 $\mathrm{msec}$, respectively). ${ }^{2}$ The second line contains the number of trials at each stimulus value for which the $r$ response was given, and the third line contains the number of trials at each stimulus value for which the $\bar{r}$ response was given. Multiple data sets of this form may be included in a single file by appending successive sets of three lines or, if the same stimulus values are used throughout, by appending pairs of lines with the numbers of $r$ and $\bar{r}$ responses.
If the data are stored in the file TESTDATA.IN, PMETRIC can be invoked simply by typing the command PMETRIC TESTDATA. The resulting output will be written to an ASCII file called TESTDATA.OUT.

By default, PMETRIC computes parameter estimates using both probit analysis and the Spearman-Kärber method. The values computed by the program are illustrated in Table 2, with computations performed on the data in Table 1, using both the Spearman-Kärber method (top half of table) and probit analysis (bottom half). The output includes both moment-based and percentile-based estimates (left and right sides of the table, respectively) for measures of central tendency (mean and median), dispersion (standard deviation and DL), skewness, and kurtosis. Associated with each estimate is a standard error of estimation obtained using the bootstrapping procedure (e.g., DiCiccio \& Romano, 1988; Foster \& Bischof, 1991). Note that the skewness and kurtosis measures obtained from probit analysis are determined primarily by the assumptions of the analysis, rather than by the observed data; that is, both must equal zero if the true psychometric function has the shape of a cumulative normal distribution.

The PMETRIC program has a number of analysis options that can be controlled via a separate options file. Four of these are of particular importance.

1. The data can come from either yes-no or $m$-AFC tasks.

2. The optimal probit fit can be defined either as the fit maximizing likelihood or as the one minimizing discrepancies as measured with a $\chi^{2}$ test (Guilford, 1936). For example, for the data shown in Table 1, PMETRIC computes a $\chi^{2}$ of 26.93 with $d f=8$ (i.e., 10 stimulus levels minus 2 estimated parameters $=8$ ). This observed $\chi^{2}$ value is highly significant at $p<.001$ and, thus, suggests that the normal distribution is not suited to summarize these data. In such a situation, a user might prefer the Spearman-Kärber method for analyzing an observed psychometric function.

3. A probit-like analysis can be carried out using many assumed underlying distributions other than a normal. For example, in a particular experiment, there might be good theoretical reasons to suspect that the psychometric function would have the shape of a cumulative Weibull distribution rather than a normal (e.g., Green \& Luce, 1975; Nachmias, 1981; Quick, 1974). In that case, PMETRIC could be instructed to use the Weibull, rather than the normal, in fitting the psychometric function. For an additional illustration, we analyzed the data shown in Table 1, assuming an underlying Weibull distribution. Under this distributional assumption, PMETRIC provides the following estimates: mean $=881.26$, median $=885.78$, $S D=67.21$, and DL $=45.66$. The observed $\chi^{2}$ for this distribution is 23.46 , with $d f=7$. This value is again significant ( $p<.001)$, and thus the Weibull, like the normal, provides an unacceptable fit. A more complete list of probability distributions that can be specified for probit analysis within PMETRIC, along with their parameters, 
Table 2

Sample Output for the Data in Table 1

\begin{tabular}{|c|c|c|c|c|c|}
\hline \multicolumn{3}{|c|}{ Moment-Based } & \multicolumn{3}{|c|}{ Percentile-Based } \\
\hline Parameter & Estimate & $S E$ & Parameter & Estimate & $S E$ \\
\hline \multicolumn{6}{|c|}{ Parameters Estimated Using the Spearman-Kärber Method } \\
\hline Measures of location & & & & & \\
\hline Mean & 880.09 & 4.44 & Median & 870.00 & 7.17 \\
\hline $\begin{array}{l}\text { Measures of dispersion } \\
\quad S D\end{array}$ & 65.74 & 3.62 & DL & 44.15 & 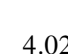 \\
\hline Measures of skewness & & & & & \\
\hline$E\left[(X-\text { mean })^{3}\right] / S D^{3}$ & 0.34 & 0.25 & $\left(q_{3}-2 \cdot q_{2}+q_{1}\right) /\left(q_{3}-q_{1}\right)$ & 0.17 & 0.12 \\
\hline$E\left[(X-\text { mean })^{3}\right]^{1 / 3}$ & 46.03 & 24.66 & $\begin{array}{l}\left(z_{9}-2 \cdot z_{5}+z_{1}\right) /\left(z_{9}-z_{1}\right) \\
\text { (Mean }- \text { Median)/SD }\end{array}$ & $\begin{array}{l}0.26 \\
0.15\end{array}$ & $\begin{array}{l}0.10 \\
0.07\end{array}$ \\
\hline Measures of kurtosis & & & & & \\
\hline$E\left[(X-\text { mean })^{4}\right] / S D^{4}$ & 3.57 & 0.67 & $\mathrm{DL} /\left(z_{9}-z_{1}\right)$ & 0.28 & 0.02 \\
\hline$E\left[(X-\text { mean })^{4}\right]^{1 / 4}$ & 90.35 & 6.19 & & & \\
\hline \multicolumn{6}{|c|}{ Parameters Estimated Using Probit Analysis } \\
\hline Measures of location & & & & & \\
\hline Mean & 880.77 & 4.51 & Median & 880.77 & 4.51 \\
\hline Measures of dispersion & & & & & \\
\hline$S D$ & 63.65 & 4.90 & DL & 42.91 & 3.30 \\
\hline Measures of skewness & & & & & \\
\hline$E\left[(X-\text { mean })^{3}\right] / S D^{3}$ & 0.00 & 0.00 & $\left(q_{3}-2 \cdot q_{2}+q_{1}\right) /\left(q_{3}-q_{1}\right)$ & 0.00 & 0.00 \\
\hline$E\left[(X-\text { mean })^{3}\right]^{1 / 3}$ & 0.00 & 0.00 & $\left(z_{9}-2 \cdot z_{5}+z_{1}\right) /\left(z_{9}-z_{1}\right)$ & 0.00 & 0.00 \\
\hline & & & $($ Mean - Median) $/ S D$ & 0.00 & 0.00 \\
\hline Measures of kurtosis & & & & & \\
\hline$E\left[(X-\text { mean })^{4}\right] / S D^{4}$ & 3.00 & 0.00 & $\mathrm{DL} /\left(z_{9}-z_{1}\right)$ & 0.26 & 0.00 \\
\hline$E\left[(X-\text { mean })^{4}\right]^{1 / 4}$ & 83.77 & 6.44 & & & \\
\hline Measures of fit & & & & & \\
\hline Ln(Likelihood) & -221.07 & 15.58 & Chi-square & 26.93 & 16.39 \\
\hline Chi-square $d f$ & 8.00 & & $\operatorname{Pr}($ Chi-square $)$ & .001 & 0.25 \\
\hline
\end{tabular}

Note-These parameter estimates were computed from the data shown in Table 1, using the Spearman-Kärber method and probit analysis, as indicated. Moment-based estimates are shown on the left side of the table and percentile-based estimates are shown on the right. Associated with each estimate is a standard error $(S E)$ computed using 200 bootstrap samples. $S D$ and DL are the dispersion parameters standard deviation and difference limen, respectively. $q_{i}$ is the $i$ th quartile of the distribution, and $z_{i}$ is the $i$ th decile.

can be found in the documentation for the program CUPID (J. Miller, 1998), although a number of new distribution options have been added since that report.

4. The user can specify the number of bootstrap samples used to obtain the standard errors of the estimates, thereby choosing an appropriate point on the tradeoff between computer time and accuracy of results.

In addition to the program PMETRIC.EXE, PMETRIC comes with a program called PMETGEN.EXE that can be used to generate psychometric function data for simulation research and with manuals for both programs in public document format (PDF). The complete versions can be obtained by sending a 3.5-in. high-density floppy disk and a stamped, self-addressed international mailer to the first author, and they are also available by ftp from common archive sites in the file PMETRIC1_2.ZIP (the numbers in the file name may change as future versions are developed).

\section{Using PMETRIC to Compare Experimental Conditions}

In psychophysical work, researchers most often compare psychometric functions across two or more experimental conditions to isolate the effects of an experimen- tal manipulation. Although PMETRIC fits psychometric functions one at a time, it can be used to compare psychometric functions across conditions in at least two types of studies.

In multiparticipant studies, a single psychometric function is generated for each participant in each experimental condition. PMETRIC can be used to estimate the parameters of each such function, and these parameters can be compared across conditions, using conventional statistical tests (e.g., a $t$ test or an analysis of variance [ANOVA] ). For example, Yeshurun and Carrasco (1999) investigated whether a shift of spatial attention toward a peripheral stimulus improved perceptual resolution for this stimulus. In an experimental condition, the stimulus appeared at an attended location; in a control condition, it appeared at an unattended location. The psychometric function obtained for each participant in each condition was fitted with a parametric model, and a single measure (i.e., the DL) was derived from each fitted function. The values of this dependent measure were then submitted to a conventional paired $t$ test to assess whether spatial attention improved discrimination performance, on average, across participants. The output of PMETRIC could be used to compare conditions in an analogous study, 
using either dependent measures assessed via $t$ tests or ANOVAs across participants on either parametric or nonparametric (i.e., Spearman-Kärber) estimates obtained from the observed psychometric functions.

Alternatively, in single-participant studies, each observer is tested more extensively, and the researcher is interested in assessing the effect of an experimental manipulation at the level of an individual observer. In such studies, it is obviously inappropriate to use $t$ tests or ANOVAs across participants. It would often still be possible, however, to use one of these conventional procedures on estimates obtained from multiple subsets of the participant's data. For example, estimates may be obtained from the observed psychometric function separately for each day of testing, and a conventional test could be used with days, rather than participants, serving as replications. Alternatively, if it is undesirable to partition the participant's data into subsets, comparisons of experimental conditions can be made by computing confidence intervals around each parameter, using the bootstrap estimate of its standard error. If the confidence intervals for the parameter in two experimental conditions overlap, one cannot reject the hypothesis that the two parameters are different. If the two intervals do not overlap, however, this hypothesis can be rejected.

\section{REFERENCES}

BLISS, C. I. (1935). The calculation of the dosage mortality curve. Annals of Applied Biology, 22, 134-167.

Church, J. D., \& CoвB, E. B. (1973). On the equivalence of the Spearman-Kärber and maximum likelihood estimates of the mean. Journal of the American Statistical Association, 68, 201-202.

DiCiccio, T. J., \& RoMANO, J. P. (1988). A review of bootstrap confidence intervals. Journal of the Royal Statistical Society: Series B, 50, 338-354.

Epstein, B., \& Churchman, C. W. (1944). On the statistics of sensitivity data. Annals of Mathematical Statistics, 15, 90-96.

ERnST, M. O., \& BANKS, M. S. (2002). Humans integrate visual and haptic information in a statistically optimal fashion. Nature, 415, 429-433.

FALMAGNE, J. C. (1985). Elements of psychophysical theory. Oxford: Oxford University Press.

FECHNER, G. T. (1860). Elemente der Psychophysik. Leipzig: Breitkopf \& Härtel.

FINNEY, D. J. (1952). Probit analysis: A statistical treatment of the sigmoid response curve (2nd ed.). Cambridge: Cambridge University Press.

FINNEY, D. J. (1978). Statistical method in biological assay. London: Charles Griffin

Flanagan, J. R., \& Bandomir, C. A. (2000). Coming to grips with weight perception: Effects of grasp configuration on perceived heaviness. Perception \& Psychophysics, 62, 1204-1219.

Foster, D. H., \& BISCHOF, W. F. (1987). Bootstrap variance estimators for the parameters of small-sample sensory-performance functions. Biological Cybernetics, 57, 341-347.

Foster, D. H., \& BISCHOF, W. F. (1991). Thresholds from psychometric functions: Superiority of bootstrap to incremental and probit variance estimators. Psychological Bulletin, 109, 152-159.

Gescheider, G. A. (1997). Psychophysics: The fundamentals(3rd ed.). Hillsdale, NJ: Erlbaum.

GREEN, D. M., \& LuCE, R. D. (1975). Parallel psychometric functions from a set of independent detectors. Psychological Bulletin, 82, 483-486.
GRONDIN, S. (2001a). Discriminating time intervals presented in sequences marked by visual signals. Perception \& Psychophysics, 63, 1214-1228.

Grondin, S. (2001b). From physical time to the first and second moments of psychological time. Psychological Bulletin, 127, 22-44. GUILFORD, J. P. (1936). Psychometric methods. New York: McGraw-Hill. KaERnBACH, C. (1991). Simple adaptive testing with the weighted up-down method. Perception \& Psychophysics, 49, 227-229.

KÄRBER, G. (1931). Beitrag zur kollektiven Behandlung pharmakologischer Reihenversuche [A contribution to the collective treatment of a pharmacological experimental series]. Archiv für experimentelle Pathologie \& Pharmakologie, 162, 480-483.

KLEIN, S. A. (2001). Measuring, estimating, and understanding the psychometric function: A commentary. Perception \& Psychophysics, 63, 1421-1455.

Klein, S. A., \& Macmillan, N. A. (Eds.) (2001). Psychometric functions and adaptive methods. Perception \& Psychophysics, 63(8).

LEEK, M. R. (2001). Adaptive procedures in psychophysical research. Perception \& Psychophysics, 63, 1279-1292.

Lieberman, H. R. (1983). Computation of psychophysical thresholds using the probit technique. Behavior Research Methods \& Instrumentation, 15, 446-448.

Macmillan, N. A., \& Creelman, C. D. (1991). Detection theory: A user's guide. Cambridge: Cambridge University Press.

MARKS, L. E., \& GESCHEIDER, G. A. (2002). Psychophysical scaling. In H. Pashler \& J. Wixted (Eds.), Stevens' handbook of experimental psychology: Vol. 4. Methodology in experimental psychology (3rd ed., pp. 91-138). New York: Wiley.

MiLleR, G. A., \& GARNER, W. R. (1944). Effect of random presentation on the psychometric function: Implications for a quantal theory of discrimination. American Journal of Psychology, 57, 451-467.

MiLler, J. (1998). Cupid: A program for computations with probability distributions. Behavior Research Methods, Instruments, \& Computers, 30, 544-545.

MILLER, J., \& ULRICH, R. (2001). On the analysis of psychometric functions: The Spearman-Kärber method. Perception \& Psychophysics, 63, 1399-1420.

NACHMIAS, J. (1981). On the psychometric function for contrast detection. Vision Research, 21, 215-223.

QUICK, R. F. (1974). A vector magnitude model of contrast detection. Kybernetik, 16, 65-67.

RAMMSAYER, T., \& UlRich, R. (2001). Counting models of temporal discrimination. Psychonomic Bulletin \& Review, 8, 270-277.

Rose, D., \& Summers, J. (1995). Duration illusions in a train of visual stimuli. Perception, 24, 1177-1187.

SPEARMAN, C. (1908). The method of "right and wrong cases" ("constant stimuli") without Gauss's formulae. British Journal of Psychology, 2, 227-242.

SPENCE, C., Shore, D. I., \& Klein, R. M. (2001). Multisensory prior entry. Journal of Experimental Psychology: General, 130, 799-832.

STERnBerG, S., \& KNOLL, R. L. (1973). The perception of temporal order: Fundamental issues and a general model. In S. Kornblum (Ed.), Attention and performance IV (pp. 629-685). New York: Academic Press.

SternberG, S., Knoll, R. L., \& Zukofsky, P. (1982). Timing by skilled musicians. In D. Deutsch (Ed.), The psychology of music (pp. 181-239). New York: Academic Press.

Ste Vens, S. S., Morgan, C. T., \& Volkmann, J. (1941). Theory of the neural quantum in the discrimination of loudness and pitch. American Journal of Psychology, 54, 315-335.

Strasburger, H. (2001a). Converting between measures of slope of the psychometric function. Perception \& Psychophysics, 63, 13481355.

STRASBURGER, H. (2001b). Invariance of the psychometric function for character recognition across the visual field. Perception \& Psychophysics, 63, 1356-1376.

UlRICH, R., \& Miller, J. O. (in press). Threshold estimation in twoalternative forced-choice (2AFC) tasks: The Spearman-Kärber method. Perception \& Psychophysics. 
WiCKENS, T. D. (2001). Elementary signal detection theory. Oxford: Oxford University Press.

Woodworth, R. S., \& SCHLOSBERG,H. (1954). Experimental psychology. New York: Holt.

YeSHURUN, Y., \& CARRASCO, M. (1999). Spatial attention improves performance in spatial resolution tasks. Vision Research, 39, 293-306.

\section{NOTES}

1. When an observed psychometric function is nonmonotonic due to binomial variability, however, it should be monotonized before applying the Spearman-Kärber method. An algorithm for this purpose is described in J. O. Miller and Ulrich (2001), and a computer program for this algorithm is provided by Klein (2001). PMETRIC automatically applies this algorithm to an observed psychometric function when it is nonmonotonic.

2. An important limitation of the Spearman-Kärber procedure arises when the observed psychometric function has been truncated-that is, when the observed probabilities for the most extreme stimulus levels differ from 0.00 and 1.00 (cf. Woodworth \& Schlosberg, 1954, p. 209). In that case, the estimates provided by the Spearman-Kärber method depend on the values assumed for the lower and upper bounds of the psychometric function. Thus, the Spearman-Kärber method must be used with caution in this situation.

(Manuscript received December 7, 2001; revision accepted for publication May 27, 2003.) 